Influence of tetraalkyl ammonium ions on the structure of poly (rG-dC) • poly (rG-dC): unexpected transitions among the $\mathrm{Z}, \mathrm{A}$ and $\mathrm{B}$ conformations

Sumedha D.Jayasena and Michael J.Behe

Department of Chemistry, Lehigh University, Bethlehem, PA 18015, USA

Received October 2, 1986; Revised and Accepted April 9, 1987

\begin{abstract}
The conformation of the double-stranded, mixed ribodeoxyribo polynucleotide, poly (rG-dC) poly ( $r G-d C$ ), has been examined in the presence of tetraalkyl ammonium ions. Tetramethyl ammonium ion stabilizes the "low salt" $\mathrm{Z}$ conformation (1) of the polymer from submillimolar to molar concentrations of the counterion. In the presence of tetraethyl and tetrapropyl ammonium lons the polymer exists in the low salt $z$ form up to 2 $m M$ concentration of the counterions and then flips to the right hand helical A form. With tetrabutyl ammonium counterions the polymer is in an $A$ conformation at low ion concentrations and converts to $a$ B form at concentrations greater than thirty millimolar. These results are interpreted in terms of electrostatic and solvent interactions of the polynucleotide.
\end{abstract}

\title{
INTRODUCTION
}

An understanding of parameters affecting the conformation of polynucleotides is necessary to understand the function of nucleic acids in vivo. An important factor affecting polynucleotide conformation is the type of counterion associated with the charged polymer. A particularly interesting interaction, between nucleic acids and tetraalkyl ammonium ions, has been studied by several laboratories (2-5) over the years. It was observed that tetramethyl ammonium ion causes sharpening of the melting transitions of heterogeneous genomic DNA (3) and that the counterion stabilizes synthetic poly (dA-dT) poly (dA-dT) against melting when compared to $\mathrm{Na}^{+}$as a counterion (4). An explanation offered for this is that tetraalkyl ammonium lons preferentially bind AT base pairs (3).

In the present study we examine the effect of tetraalkyl ammonium ions on poly ( $\mathrm{rG}-\mathrm{dC}$ ) poly (rG-dC), a polymer containing $50 \%$ ribose and $50 \%$ deoxyribose residues. This polymer was 
previously shown to undergo two right-left hand helical transitions at different concentrations of $\mathrm{NaCl}$ : an $\mathrm{A}$ to high salt $z$ transition and an $A$ to low salt $z$ transition (1). The presence of moderate concentrations of tetraalkyl ammonium ions causes a re-ordering of transitions of the polymer, and an additional conformational transition, to a $B$ form, is observed. The results are discussed in terms of the electrostatic and solvent interactions of the polymer.

\section{YATERTATS AND METHODS}

\section{Synthesis of the Polymer}

E. coli DNA polymerase I large fragment (Klenow fragment) was purchased from United states Biochemical Corporation. Poly (dI-dC) poly (dI-dC) was obtained from P-I Biochemicals. rGTP and dCTP were purchased from sigma.

The polymer was synthesized as previously described (1). The reaction medium contained $40 \mathrm{mM} N, \mathrm{~N}$-bis(2-hydroxyethyl)glycine

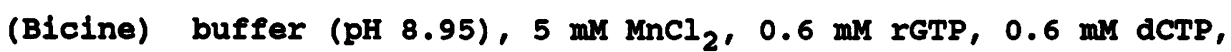
$100 \mu \mathrm{g}$ of poly (dI-dC) poly (dI-dC), and 20 units of klenow fragment in $1 \mathrm{ml}$. After a $16 \mathrm{hr}$ incubation at $37^{\circ} \mathrm{C}$, the entire mixture was added to $20 \mathrm{ml}$ of reaction medium containing the same reactants in the same concentrations except that no further poly (dI-dc) poly (dI-dC) was added. After a second $16 \mathrm{hr}$ incubation at $37^{\circ} \mathrm{C}$, the polymer was precipitated with ethanol, resuspended in $0.4 \mathrm{ml}$ of $10 \mathrm{mu} \mathrm{Tris}-\mathrm{HCl}$ ( $\mathrm{pH} \mathrm{7.5)}$, extracted twice with phenol and chloroform, and passed through a column of sephadex G-50 equilibrated with $10 \mathrm{mM}$ Tris-HCl $\mathrm{pH} \mathrm{7.5/0.1} \mathrm{mM} \mathrm{EDTA.} \mathrm{The} \mathrm{total}$ yield was about $2 \mathrm{mg}$ of the polymer, which was shown to be 600-1000 base pairs long by gel electrophoresis.

Prior to a spectroscopic measurement, polynucleotide was passed through a column of sephadex G-50 equilibrated with the solution for the initial measurement.

\section{Tetraalkyl ammonium solutions}

Tetraalkyl ammonium hydroxides were purchased from Aldrich Chemical company and stock solutions were titrated to neutrality with concentrated HCl. stock solutions of EDTA were made by titrating EDTA (free acid, Aldrich chemical Co.) with a tetraalkyl ammonium hydroxide solution. 


\section{Spectroscopic studies}

Circular dichroic spectra were recorded on a Jasco J-500A spectropolarimeter interfaced with an IBM-XT microcomputer. Typically 10 spectra were recorded and averaged to increase the signal to noise ratio. Before each spectrum was recorded the polymer was equilibrated at room temperature for 30 minutes with tetraalkyl ammonium ion at the desired concentration. spectra were obtained at ambient temperature.

\section{RESULTS}

The far ultraviolet CD spectrum of poly (rG-dC) poly (rG-dC) in the presence of [tetramethyl ammonium ion] ranging from $0.5 \mathrm{mM}$ to $3 M$ is very similar to that for the $Z$ form of the polynucleotide previously seen in solutions of very low or very high [NaCl] (1). To be certain that the CD spectrum did reflect a $z$ form the circular dichroism spectrum of the polymer was recorded down to $180 \mathrm{~nm}$. As seen in Fig. 1 a large negative peak at $193 \mathrm{~nm}$, indicative of a $\mathrm{Z}$ form polymer (6), is obtained.

The behavior of the polymer in the presence of tetraethyl and tetrapropyl ammonium counterions is shown in Fig. 2. In both

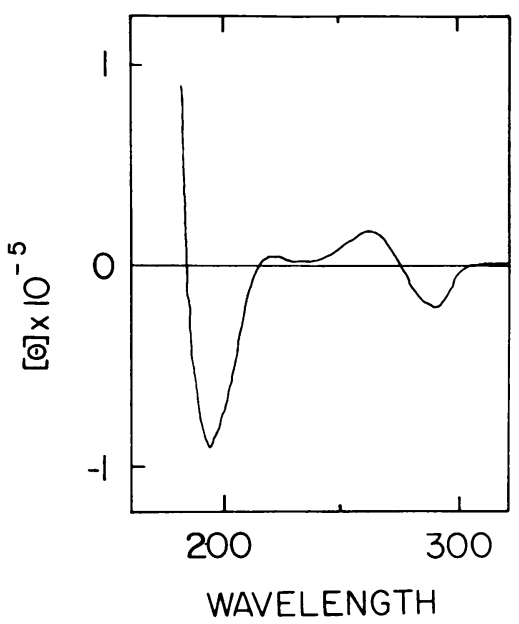

Fig. 1- Circular dichroism spectrum down to $180 \mathrm{~nm}$ of poly (rG-dC) poly (rG-dC) in $20 \mathrm{mM}$ tetramethyl ammonium chloride. 


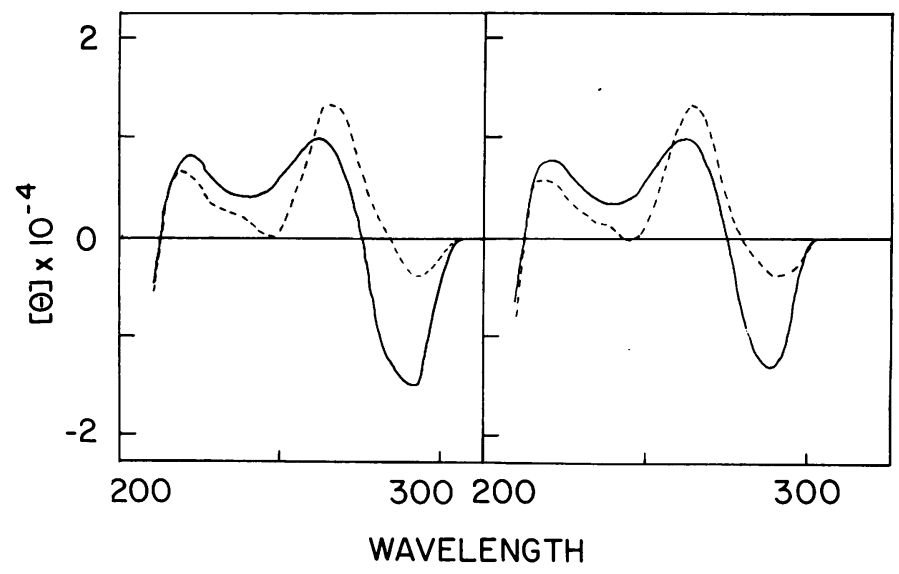

Fig. 2- Circular dichroism spectrum of poly (rG-dC) poly (rG-dC) in the presence of: left panel- $1 \mathrm{mM}$ ( or $10 \mathrm{mM}$ (- - - , A form) tetraethyl ammonium chloride; right panel- $1 \mathrm{mM}($, $\mathrm{Z}$ form) or $10 \mathrm{mM}$ ( - - - , A form) tetrapropyl ammonium chloride. All solutions contained $0.1 \mathrm{mM}$ EDTA.

cases poly ( $r G-d C$ ) poly ( $r G-d C$ ) gives a $Z$ form $C D$ spectrum if the concentration of counterion is less than $2 \mathrm{mM}$. Above $5 \mathrm{mM}$ the $C D$ spectrum is that for a canonical A form polymer. At higher counterion concentrations, up to $I M$, the $C D$ spectrum remains the same.

In the presence of tetrabutyl ammonium counterions, poly (rG-dC) poly ( $r G-d C$ ) gives an $A$ form $C D$ spectrum even at the lowest concentration of counterion examined $(0.5 \mathrm{mM})$. As the concentration of counterion is increased from 30 to $80 \mathrm{mM}$ a transition takes place (Figs. $3 \& 4$ ). The polymer now gives a CD spectrum very similar to that of a typical B form polydeoxynucleotide, even though the polymer consists of 508 ribonucleotide residues. Increasing the tetrabutyl ammonium ion concentration up to $1 \times$ results in no further changes in the CD spectrum. However, if mothanol is included in the solution, in the presence of $400 \mathrm{mM}$ tetrabutyl ammonium ion the CD spectrum cooperatively reverts from B form to that of an A form polymer (data not shown). The midpoint of the transition is $\sim 258$ methanol. 


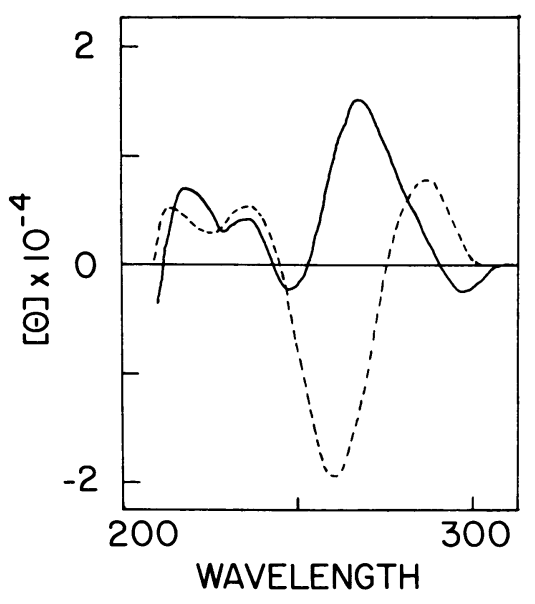

Fig. 3- Circular dichroism spectrum of poly (rG-dC) poly (rG-dC) in the presence of $10 \mathrm{mM}$ ( , A form) or $100 \mathrm{mM}$ ( - - - , B form) tetrabutyl ammonium chloride, $0.1 \mathrm{mM}$ EDTA.

\section{DISCUSSION}

Effect of tetraalkylammonium ions on the conformation of poly $(r G-d C) \cdot$ poly $(r G-d C)$

We observed earlier (7) that methylated poly (rG-dc) poly (rG-dC) showed a counterion-dependent conformational transition. Although the polymer was in the $A$ conformation in the presence of $5 \mathrm{mM}$ Tris buffer, it converted to a low salt $\mathrm{z}$ conformation in the presence of $5 \mathrm{mM}$ triethanolamine buffer. Reasoning that triethanolamine caused the transition because it was a tertiary amine and therefore less likely to be an effective screening agent of DNA phosphate charge, we decided to look at the effect of tetraalkyl ammonium ions, which are even larger, bulkier, and presumably less effective counterions. Tetraalkyl ammonium ions have been known for some time to bind preferentially to AT rich polymers of DNA (2) and have been used to abolish the preferential melting of AT versus GC base pairs of DNA at molar counterion concentrations (3). However, the synthetic polymer studied here contains no AT base pairs and we observe effects at much lower levels of counterion. 


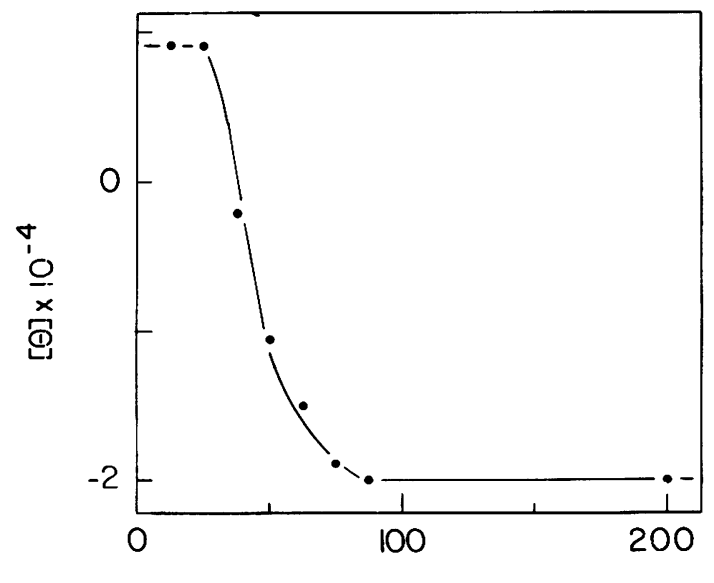

[TBA], mM

Fig. 4- Molar ellipticity of poly (rG-dC) poly (rG-dC) at $260 \mathrm{~nm}$ as a function of tetrabutyl ammonium concentration.

As described in the Results section the smallest counterion examined, tetramethyl ammonium, causes poly (rG-dC) poly (rG-dC) to remain in a low salt $z$ conformation over a wide range of counterion concentrations. This was the expected result. The fact that the A conformation of the polymer was not induced even by molar concentrations of tetramethyl ammonium ion was ascribed to the inability of the quaternary amine to screen the higher phosphate charge repulsion of the A form. A likely reason for this is that the large size of the tetramethyl ammonium ion could prevent it from condensing $(8)$ on the polynucleotide to the same degree that a smaller cation could.

The subsequent results with tetraethyl and tetrapropyl ammonium lons did not follow along the same lines. We expected these lons also to support the low salt $\mathrm{z}$ form at high counterion concentrations, or perhaps to destabilize the polynucleotide to the point of melting, due to their larger size compared to tetramethyl ammonium 1on. Instead, as shown in Fig. 2, both ions caused the transition of the polymer from the low salt $z$ form to the A form at about 2-5 mM counterion concentration, more efficientiy than the primary amine Tris (1). Tetrabutyl ammonium ion, the largest counterion we tested, would not support the low 
salt $Z$ form of the polymer even at the lowest counterion concentrations examined $(0.5 \mathrm{mM})$, where it was seen to already be in the $A$ form. As the concentration of tetrabutyl ammonium ion was increased past $30 \mathrm{mx}$, an apparent transition to $a$ conformation was observed.

clearly a second factor besides effectiveness of the tetraalkyl ammonium ion as a phosphate screening agent is involved in the influence of these counterions on the conformation of poly (rG-dC) poly (rG-dC). A likely possibility in an aqueous solution is the manner of interaction of the polymer-counterion system with water. It is possible that both electrostatic effects and solvent interactions influence the conformational equilibria of poly ( $r G-d C$ ) poly (rG-dC) in the following way. Tetramethyl ammonium ion causes the polymer to remain in the low salt $z$ conformation because it is an ineffective charge screening agent. The transition of the polymer to conformations with higher charge densities is not supported. with tetraethyl and tetrapropyl ammonium ions the second factor comes into play. Hydrophobic groups, like the ethyl and propyl chains of the counterions, cause ordering of surrounding water molecules (9), which may drive the polynucleotide into a conformation that has more ordered solvent molecules associated with it. If this is true then the low salt $z$ form of poly (rG-dC) poly (rG-dC) is less hydrated than the A conformation of the polymer.

The trend is continued with the next member of the series, tetrabutyl ammonium ion. This bulky, hydrophobic ion does not support the low salt $z$ conformation of poly (rG-dC) poly (rG-dC) at all. As seen in Figs. $3 \& 4$, the polymer starts out in the $A$ form at low tetrabutyl ammonium ion concentrations, and a transition to the $B$ form occurs above $30 \mathrm{mM}$ counterion. The $B$ conformation is known to be more highly hydrated than either the $A$ or the $z$ forms (10). Thus the counterion with the largest hydrophobic groups, which cause the most ordering of solvent, drives the polymer into the most hydrated conformation. The thesis that water is important in driving the A-B transition is supported by the observation that if the polymer in the B form is subjected to dehydrating conditions, such as the presence of 
significant concentrations of methanol (see Results), a cooperative transition from the $B$ to the A conformation takes place.

A polynucleotide containing $50 \%$ ribonucleotides in the $B$ form

Assignment of polynucleotide conformations on the basis of circular dichroism studies has sometimes given misleading results $(11-13)$. In those instances the polymer under consideration was either in solutions of extremely high salt concentrations, organic solvents, or it was covalently modified. Those situations do not occur here and, although circular dichroism spectra do not prove structure, there are several reasons to think that the spectrum shown in Fig. 3 for poly (rG-dC) poly (rG-dC) in solutions of tetrabutyl ammonium ion concentration greater than $80 \mathrm{mM}$ does reflect the polymer's occurrence in a B conformation. 1) The spectrum is very similar to that for the $B$ conformation of the fully deoxy polymer poly (dG-dC) poly (dG-dC) both in the location and intensities of peaks. 2) The spectrum is well-behaved in that there is no CD signal tailing out beyond 300 $\mathrm{nm}$, as in some covalently modified or aggregated polymers. 3) The spectrum was recorded for an aqueous solution of moderate ionic strength. 4) The CD spectrum below $30 \mathrm{mM}$ tetrabutyl ammonium concentration is that for the A form of the polymer, similar to spectra seen in the presence of tetraethyl and tetrapropyl ammonium and alkali metal ions (1), so that the mere presence of tetrabutyl ammonium ions does not disturb the $C D$ spectrum. 5) The transition seen in Fig. 5 is smooth and cooperative, as expected for a transition between different polynucleotide conformations. Thus we feel it is reasonable to conclude that the conformation of poly ( $r G-d C$ ) poly ( $r G-d C$ ) above $80 \mathrm{mM}$ tetrabutyl ammonium ion concentration is a $B$ form.

The ability of poly (rG-dC) poly (rG-dC), a polymer containing 508 ribonucleotide residues, to convert to $a$ B conformation is surprising. Although it was shown earlier that a hydrated fiber of poly rA poly dT could exist in a B form (14), it is known that synthetic polydeoxynucleotides containing exclusively $A \cdot T$ base pairs are very stable in the $B$ form due to the preferential interaction of $A \cdot T$ base pairs in that conformation with solvent to form a water "spine" in the minor 
groove (15). No such interaction stabilizes $G \cdot C$ base pairs in the $B$ form and, experimentally, polymers containing exclusively $G \cdot C$ base pairs readily convert to the $A$ conformation under appropriate solvent conditions.

The experiment with fibrous poly rA.poly dT (14) showed that a double stranded polynucleotide could exist in the B form with one strand consisting entirely of ribonucleotides. The experiments reported here demonstrate that a synthetic polynucleotide containing ribonucleotides on both strands can also exist in the $B$ conformation. It seems a logical extension of these results to conclude that other RNA.DNA hybrids or even double stranded RNA could exist in a B conformation under highly hydrating conditions. Whether this ever occurs in vivo during transcription or in double stranded or hairpin regions of RNA is presently unknown.

\section{ACKNOWLEDGMENTS}

This work was supported by grants from the National Institutes of Health (GM36343) and the National Science Foundation (DMB-8643013). M.B. is a recipient of a Research Career Development Award from the National Institutes of Health (CA01159).

\section{REFERENCES}

1. Wu, H. Y. \& Behe, M.J. (1984) Prof. Natl. Acad. Sci. USA 81, 7284-7287.

2. Shapiro, J.T., Stannard, B.S. \& Felsenfeld, G. (1969) Blochemistry 8, 3233-3241.

3. Melchior, W.B. \& von Hippel, P.H. (1973) Proc. Natl. Acad. Sci. USA 70, 298-302.

4. Marky, L.A., Patel, D. \& Breslauer, K.J. (1981) Biochemistry 20, 1427-1431.

5. Wood, W.I., Gitschier, J., Lasky, L.A. \& Lawn, R.M. (1985) Proc. Natl. Acad. Sci. USA 82, 1585-1588.

6. Sutherland, J.C., Griffin, K.P., Keck, P.C. \& Takacs, P.Z. (1981) Proc. Natl. Acad. Sc1. USA 78, 4801-4804.

7. Wu, H. Y. \& Behe, M.J. (1985) Nucleic Acids Res. 13, 3931-3940.

8. Manning, G. (1978) Q. Rev. Biophys. 11, 179-245.

9. Tanford, C. (1978) Science 200, 1012-1018.

10. Dickerson, R.E., Drew, H.R., Conner, B.N., Wing, R.M., Fratin1, A.V. \& Kopka, M.I. (1982) Science 216, 475-485.

11. Zimmerman, S.B. \& Pheiffer, B. (1980) J. Mol. B101. 142, 315-330. 
12. Tomasz, M., Barton, J.K., Magliozzi, C.C., Tucker, D., Lafer, E.M. \& Stollar, B.D. (1983) Proc. Natl. Acad. Sci. USA 80, 2874-2878.

13. Chen, C.Y., Pheiffer, B.H., Zimmerman, S.B. \& Hanlon, S. (1983) Biochemistry 22, 4746-4751.

14. Zimmerman, S.B. \& Pheiffer, B.H. (1981) Proc. Natl. Acad. Sci. USA 78, 78-82.

15. Kopka,M.L., Fratini, A.V., Drew, H.R. \& Dickerson, R.E. (1983) J. Mol. Biol. 163, 129-146. 